Patricia R. Allaire and Robert E. Bradley

\section{Geometric Approaches to Quadratic Equations from Other Times and Places}

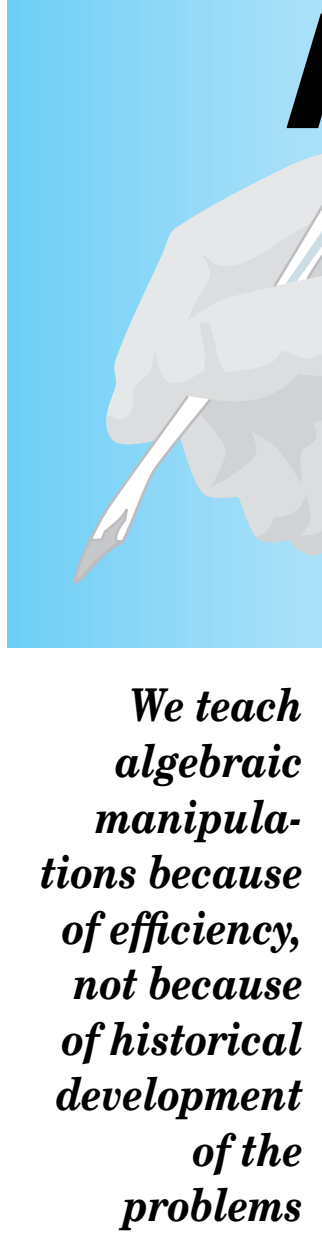

s mathematics evolves, the techniques that survive are those that have the greatest power and generality. The reason that we teach symbolic algebraic manipulation has everything to do with its efficiency and nothing to do with the historical development of the problems being solved.

We can consider the following problem:

A field has a perimeter of 40 yards and an area of 96 square yards. What are the dimensions of this field?

A modern solution would involve two variables, one of which is eliminated to derive the quadratic equation

$$
x^{2}-20 x+96=0 .
$$

This equation is then solved by factoring or by using the quadratic formula.

This procedure would seem arbitrary to someone who did not know modern algebraic techniques. The skills involved, that is, representing quantities by letters and performing algebra on these symbols, seem to have little relevance to the practical problem of finding the dimensions of a physical object. We teach these skills because of their broad applicability not only to quadratic problems but also to linear and cubic problems, as well as to problems involving rational functions, square roots, and more complicated forms.

In this article, we concentrate exclusively on quadratic problems, that is, on problems whose twentieth-century solution involves setting up a quadratic equation in one unknown. Before symbolic manipulation became the lingua franca of mathematics, mathematicians could solve quadratic problems. Their techniques usually involved some geometric feature of the situation.

Even if we admit the greater efficiency of the symbolic approach, many good reasons remain for studying geometric solutions of the quadratic. For those who are visually, as opposed to symbolically, oriented, studying geometric solutions can make both the problems themselves and the algebra involved more meaningful. Even those who are comfortable with a symbolic conception of algebra will probably find different points of view to be enriching. In addition, a historical survey of geometric techniques can be used to introduce both completing the square-it even explains the origin of the name-and deriving the quadratic formula. Finally, our classroom experience suggests that a historical approach always makes the subject more appealing to the typical student.

What follows is a collection of geometric techniques from ancient Babylonia, classical Greece, medieval Arabia, and early modern Europe that can enhance the quadratic-equation portion of an algebra course. The purpose is not to replace the symbolic approach but to offer alternative perspectives. Although the topics are interrelated, teachers do not need to present all of them. Rather, we encourage teachers to sample and experiment with as many techniques as seem appropriate to the teaching schedule and the interests and abilities of the students.

\section{GEOMETRICAL ALGEBRA}

Geometrical algebra is the branch of mathematics that is concerned with using geometrical concepts and proofs as the underpinnings of algebraic techniques. Although the ideas of geometrical algebra were clearly present in Asia, Mesopotamia, and Egypt centuries earlier, classical Greek mathematicians are usually credited with its development. When we geometrically solve a quadratic equation, we are doing geometrical algebra.

In the world of geometrical algebra, simple quantities, whether fixed numbers or unknowns, are represented by physical objects, almost always a line segment whose length, relative to some fixed unit, is the quantity in question. A product of two quantities is thus interpreted as the area of a rectangle, and a product of three quantities is interpreted as the volume of a right rectangular prism. This interpretation is the origin of our use of the

Pat Allaire,praqb@cunyvm.cuny.edu, teaches at Queensborough Community College, Bayside, NY 11364. She is interested in the history of mathematics, especially in the development of algebra in nineteenth-century Britain, and in community theater. Rob Bradley,bradley@adelphi.edu, teaches at Adelphi University, Garden City, NY 11530. His interests include probability theory, ergodic theory, the history of mathematics, and acoustic guitars. 
words square and cube for second and third powers. Because lengths, areas, and volumes are positive quantities, geometrical algebra deals exclusively with positive numbers and positive values of unknowns. Figure 1 is an example of geometrical algebra, illustrating the identity

$$
(a+b)^{2}=a^{2}+2 a b+b^{2}
$$

for positive $a$ and $b$, and the figure could even be called a proof of the identity.

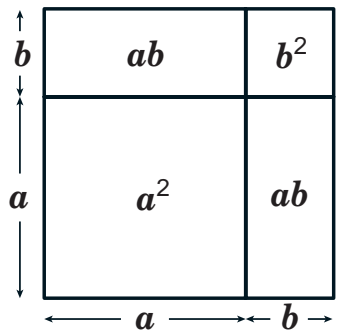

Fig. 1

An example of geometrical algebra: the identity $(a+b)^{2}=a^{2}+2 a b+b^{2}$.

A generalization of this figure can be used to illustrate the FOIL technique for multiplying two binomials. The following example is illustrated in figure 2:

$$
\begin{aligned}
(x+3)(x+2) & =x^{2}+2 x+3 x+6 \\
& =x^{2}+5 x+6
\end{aligned}
$$

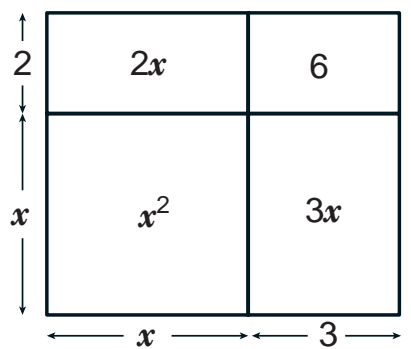

Fig. 2

$(x+3)(x+2)=x^{2}+2 x+3 x+6$

$=x^{2}+5 x+6$.

The fact that geometrical algebra deals only with positive quantities has certain implications for the way that quadratic problems are set up. For example, if we want to solve the equation $x^{2}+5 x-36=0$ geometrically, we must consider instead the form $x^{2}+5 x=36$, shown in figure 3 . If we read this equation geometrically, it asks for a quantity $x$ with the following property: when a square of side $x$ is added to a rectangle with sides of length 5 and $x$, the result is a rectangle with an area of 36 square units.

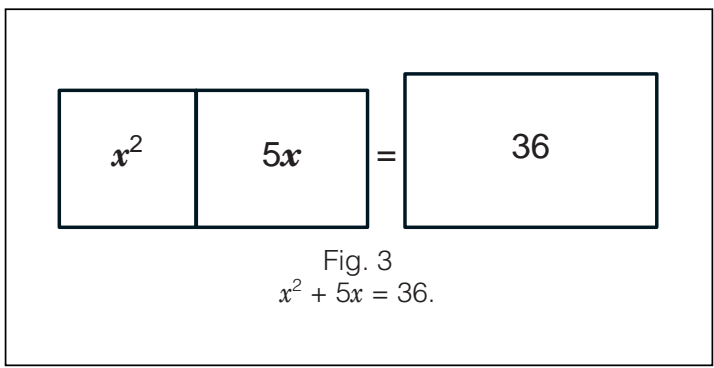

\section{CLASSIFICATION OF QUADRATIC EQUATIONS}

Geometrical algebra requires that coefficients and roots be positive, so cases must be considered

If we divide a quadratic equation by the coefficient of $x^{2}$, the equation assumes the form $x^{2}+b x+c=0$. However, since geometrical algebra by its nature requires that coefficients and roots be positive, we must work by cases when solving them geometrically. The five possible cases in which $b$ and $c$ are always positive are the following:

(1)

Because zero is not an acceptable solution in geometrical algebra, $x=b$ is the only solution in case (1). In case (2), the positive square root of $c$ is the only solution; it must be constructed in a geometrical sense before we can claim to solve this problem geometrically. We turn our attention to this construction before considering cases (3), (4), and (5).

\section{CONSTRUCTION OF $\sqrt{c}$}

In The Elements, written about 300 B.C., Euclid expressed virtually all his mathematical reasoning in a geometrical way. This work is a compilation of the basic mathematics of the Greeks beginning with Thales (ca. 625-547 B.C.). The construction shown in figure 4 is seen in The Elements (II.14). We can construct a line segment, the length of which is the square root of the length of an arbitrary line segment, given that segment and a segment of unit length, as follows:

- Construct $\overline{A B}$ with $A B=c$.

- Extend $\overline{A B}$ through $B$ to $C$ so that $B C=1$.

- Bisect $\overline{A C}$ at $M$.

- With center $M$ and radius $\overline{A M}$, construct a semicircle. 
- Erect a perpendicular to $\overline{A C}$ at $B$. Label as $E$ the point at which the perpendicular intersects the semicircle. Then $B E=\sqrt{c}$.

\section{See figure 4.}

\section{Application of areas is believed \\ to have originated with}

Pythagoras or his school

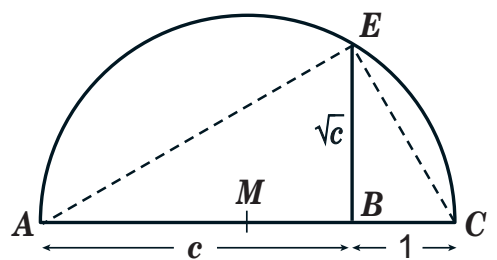

Fig. 4

Constructing a line segment, the length of which is the square root of an arbitrary line segment
We next demonstrate that this construction gives the desired length by showing that triangles $B C E$ and $B E A$ are similar, although Euclid did not use the following method of proof:

- $\angle E B C$ and $\angle E B A$ are right angles by construction and are therefore congruent.

- $\angle C E A$ is a right angle because it is inscribed in a semicircle.

- $\angle C$ and $\angle B E A$ are both complementary to $\angle C E B$, so they are congruent.

- Thus, the triangles are similar.

Since

$$
\begin{gathered}
\frac{B C}{B E}=\frac{B E}{B A}, \\
\frac{1}{B E}=\frac{B E}{c},
\end{gathered}
$$

we obtain $B E^{2}=c$ by cross multiplying. Therefore, $B E=\sqrt{c}$

\section{APPLICATION OF AREAS}

The classical Greek method for solving quadratics of types 3,4 , and 5 is called application of areas and is believed to have originated with Pythagoras (ca. 572-497 B.C.) or his school. We illustrate the technique as applied to case $3, x^{2}=b x+c$; see figure 5.

- Construct $A B$ with $A B=b$.

- Erect a perpendicular, $\overline{C B}$, to $\overline{A B}$, with $C B=\sqrt{c}$.

- Bisect $\overline{A B}$ at $M$.

- With center $M$ and radius $\overline{M C}$, construct a circle.

- Label as $D$ the point where the circle meets the extension of $\overline{A B}$ through $B$. Then $x=A D$.

A geometric justification for application of areas Why did the ancient Greeks refer to the construc-

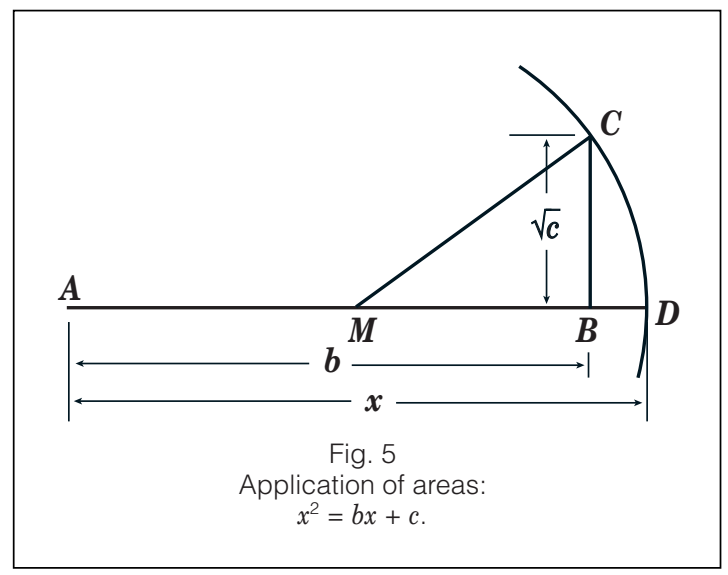

tion in figure 5 as an "application of areas"?

The term $b x$ in equation (3) represents the area of a rectangle. We are given the line segment $b$ in the setup of the problem. We "apply an area" to $b$ when we erect perpendiculars of length $x$ on it and complete a rectangle. Since the perpendiculars have length $x$, the applied area is $b x$.

To show that $x^{2}=b x+c$, we must show that this rectangle, along with another rectangle with area $c$, makes a square of side $x$, whose area is $x^{2}$. See figure 6, in which $A B$ has the given length $b$, and $\overline{A D}$

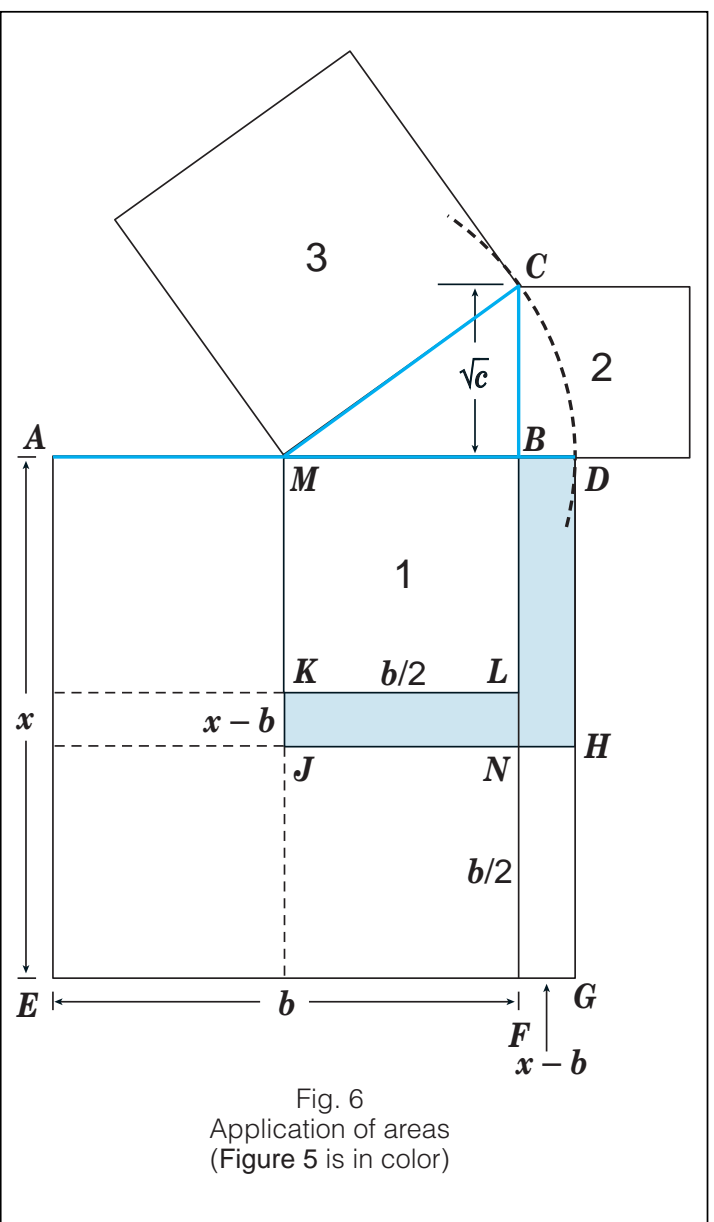


has the constructed length $x$. We erect perpendiculars $\overline{A E}$ and $\overline{D G}$, both of length $x$, to make a square $A D G E$ of area $x^{2}$. Since rectangle $A B F E$ has area $b x$, we need to show that rectangle $B D G F$ has area c. By construction, $M D=M C(C$ on $\overline{F B})$. Therefore, a square on $\overline{M D}$, such as $M D H J$, has the same area as a square on $\overline{M C}$, such as square (3).

By the Pythagorean theorem, the sum of the areas of square (1) (MBLK) and square (2) is equal to the area of square (3), which itself is equal to the area of $M D H J$. Therefore, if we subtract square $M B L K$ from square $M D H J$, the remaining piece has the same area as square (2), which is $c$, by construction.

Thus, the reverse L-shaped figure $B D H J K L$, shown shaded in figure 6, has area $c$. By picking up the rectangle $J K L N$ and moving it to the congruent rectangle NHGF, we have shown that rectangle $B D G F$ does indeed have area $c$, which is the result that we wanted.

\section{THE BABYLONIAN SOLUTION OF A QUADRATIC EQUATION}

The scribes of ancient Babylonia-an area included in modern-day Iraq-were proficient mathematicians. Extensive archaeological records indicate their achievements in the early second millennium B.C., particularly around the time of Hammurabi, about 1700 B.C. They could solve a variety of quadratic problems, the simplest being of the following form: if the sum of two numbers is 20 and the product is 96 , then what are these numbers?

This problem is a disguised version of the problem about perimeter and area that we have already encountered. When the two numbers represent the lengths of the sides of a rectangle, their sum is the semiperimeter. If two numbers $x$ and $y$ have a sum of $b$ and a product of $c$, then by substituting $y=b-x$ in the product $x y=c$, we have

$$
x^{2}+c=b x .
$$

So this problem is of type (4); any problem of type (4) can be posed in the language of areas and semiperimeters, or sums and products.

The Babylonians would have solved this problem by first guessing that $x$ equals 10 and $y$ equals 10. Since we know that $x$ and $y$ must sum to 20 , this guess is certainly reasonable. However, it cannot be correct, because a square of 10 yards on each side would have an area of 100 square yards. One approach is to subtract the correct area of 96 from this value of 100 , giving a difference of 4 square yards. Next we take the square root of 4 , which can be found geometrically, and add it to $x$. So $x=10+2$, or 12 . We take the same value, 2 , and subtract it from $y$. So $y=10-2$, or 8 . We know that this answer is correct, since $x+y=12+8$, or 20 , and $x y=12 \times 8$, or 96 .
This solution apparently does not involve any geometry. The Babylonian scribes did not use pictures to solve this problem. Instead, they used a simple rhetorical algorithm that involves manipulating the numbers 20 and 96 to obtain the dimensions. See figure 7.
1. Divide the sum $S$ in half.

2. Square the answer from part 1.

3. Subtract the product $A$ from the result in part 2. part 3.

5. Add the answer in part 4 to the answer in part 1 to determine one dimension of the field.

6. Subtract the answer to part 4 from the answer to part 1 to determine the other dimension of the field.

Fig. 7

Babylonian algorithm
4. Take the square root of the result in
$S / 2$

$(S / 2)^{2}$

$(S / 2)^{2}-A$

$\sqrt{(S / 2)^{2}-A}$

$S / 2+\sqrt{(S / 2)^{2}-A}$

$S / 2-\sqrt{(S / 2)^{2}-A}$
A geometric justification is given in figure 8: a square of side $b / 2$ has the desired semiperimeter $b$. The value $(b / 2)^{2}$ exceeds the desired area by the quantity $(b / 2)^{2}-c$, which is the area of the unshaded square of side $z$ at the upper left. The remaining shaded figure can be reassembled into a rectangle as indicated. The dimensions of the rectangle are

and

$$
x=\frac{b}{2}+\sqrt{\frac{b^{2}}{4}-c}
$$

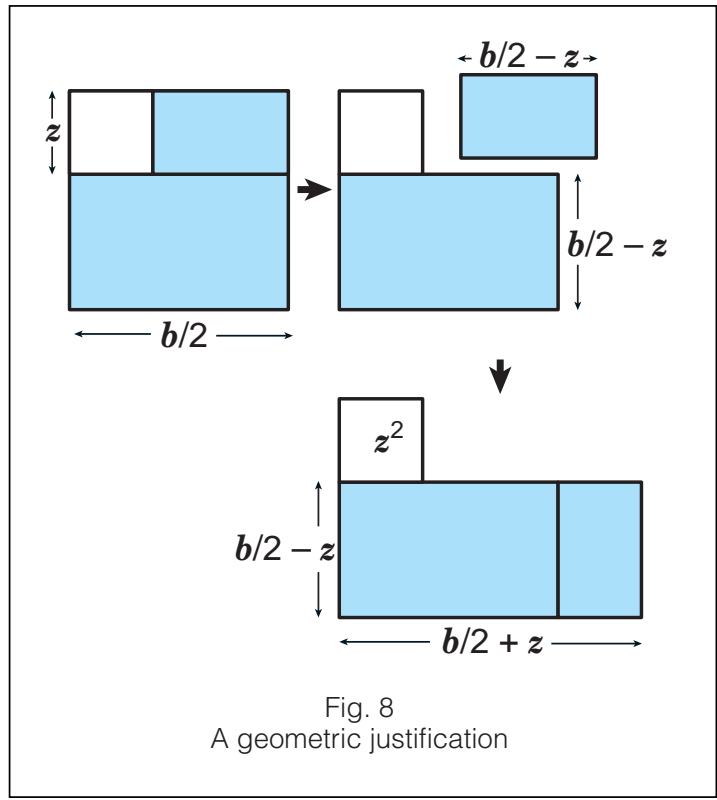




$$
y=\frac{b}{2}-\sqrt{\frac{b^{2}}{4}-c .}
$$

This procedure is practically a derivation of the quadratic formula. Because the equation that we have solved is $x^{2}-b x+c=0$ when put in standard form, no negative sign appears in front of the $b / 2$ term.

\section{AL-KHWARIZMI AND COMPLETING THE SQUARE}

In the ninth century, Muhammad ibn Musa alKhwarizmi (ca. 780-850) wrote one of the first Islamic algebra books, The Calculation of al-Jabr and al-Muqabala. The expression al-jabr in the title is the source of our word algebra. In this book on solving equations, al-Khwarizmi gave detailed explanations of the solutions of quadratic equations.

Islamic mathematics at the time of al-Khwarizmi did not accept negative numbers as either coefficients or roots; another three hundred years passed before al-Samaw'al introduced negative coefficients. Thus, algebraic solutions had to be done by cases, as we did in solving geometrically. In fact, the cases that we previously listed are those listed by al-Khwarizmi.

Al-Khwarizmi and the Islamic mathematicians used the method that we call completing the square for cases (3), (4), and (5). They used a geometric demonstration to show why this algebraic method

Islamic mathematicians used a geometric demonstration to show why the algebraic method made sense makes sense. We can see their method applied to $x^{2}+10 x=39$, an equation of type (5):

- Begin with a square of area $x^{2}$, that is, with side $x$.

- Add two rectangles, each having one side $10 / 2$, or 5 , and the other side $x$.

- The total shaded area is $x^{2}+2(5 x)$, or $x^{2}+10 x$, which is the expression on the left side of the equation. The total area is therefore 39.

- In figure 9, the upper-right corner is a square with side 5, or area 25. By adding this small square to the diagram, we "complete the square" and have a large square with area of $x^{2}+2(5 x)+$ 25 . Since we have added 25 to the original area of 39 , the area of this large square is 64 , and we can write

$$
x^{2}+10 x+25=64 .
$$

- Since the side of the large square has length $x+5$, its area is $(x+5)^{2}$. Therefore,

$$
\begin{aligned}
(x+5)^{2} & =64, \\
x+5 & =8, \\
x & =3 .
\end{aligned}
$$

Since the discussion has been in the form of geometric algebra, the process confirms only the positive value of $x$.

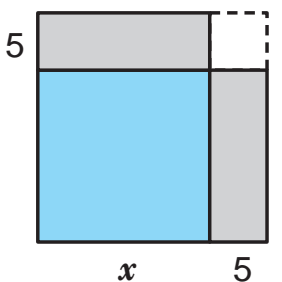

Fig. 9

Adding the small white square to the diagram "completes the square," giving $x^{2}+10 x+25=64$

\section{DESCARTES'S CONSTRUCTION}

In the seventeenth century, in the first book of $L a$ Géometrie, René Descartes (1596-1650) described a geometric method for constructing the solution of the quadratic equation $x^{2}+b x=c$. Despite its association with Descartes, this construction does not use Cartesian axes; like earlier ones, it shows the positive root only. The construction is performed as follows:

- Construct $\overline{A B}$ with $A B=\sqrt{c}$.

- Erect a perpendicular, $\overline{A C}$, to $\overline{A B}$, with $A C=b / 2$.

- Construct a circle that has center $C$ and radius $\overline{A C}$.

- Construct a line through $B$ and $C$, intersecting the circle at $E$ and $D$. Then $x=B E$.

See figure 10.

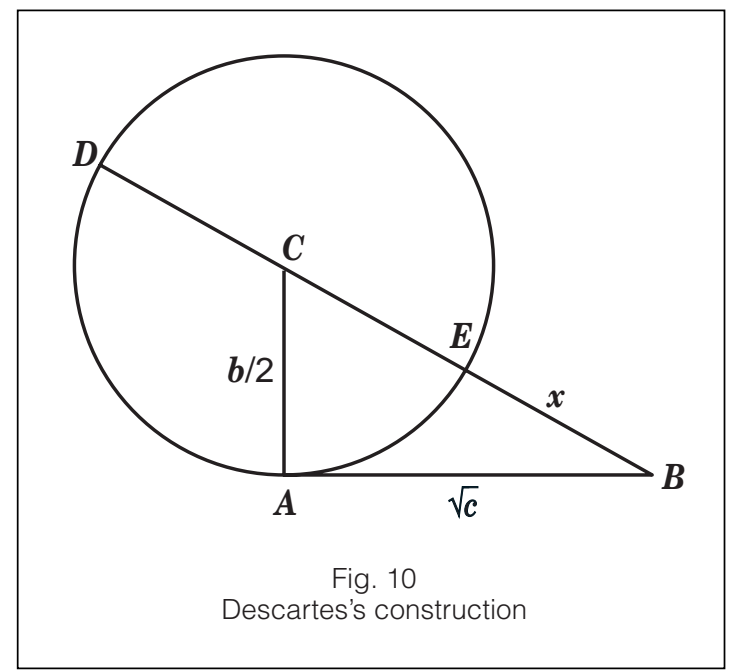

We can justify $x=B E$ by the tangent-secant theorem or, algebraically, by the Pythagorean theorem applied to triangle $A B C$.

For students who know the tangent-secant theorem-which states that if a tangent and a secant 
are drawn to a circle from an outside point, the length of the tangent is the mean proportional between the length of the secant and the length of the external segment-the verification that $x=B E$ is as follows:

$$
\begin{aligned}
\frac{B D}{A B} & =\frac{A B}{B E} \\
\frac{x+b}{\sqrt{c}} & =\frac{\sqrt{c}}{x}
\end{aligned}
$$

By cross multiplication, we find that $x(x+b)=(\sqrt{c})^{2}$, or $x^{2}+b x=c$.

An algebraic alternative to this demonstration can be given using the Pythagorean theorem. By construction, triangle $A B C$ is a right triangle. Hence,

$$
\begin{aligned}
C B^{2} & =A C^{2}+A B^{2}, \\
\left(x+\frac{b}{2}\right)^{2} & =\left(\frac{b}{2}\right)^{2}+(\sqrt{c})^{2}, \\
x^{2}+b x+\frac{b^{2}}{4} & =\frac{b^{2}}{4}+c, \\
x^{2}+b x & =c .
\end{aligned}
$$

Descartes's construction can be used to solve $x^{2}=b x+c$, in which case $x=D B$. We note the similarity between the latter construction and the solution of this equation by application of areas.

\section{CARLYLE'S METHOD}

Although Descartes's method does not use Cartesian coordinates, the following procedure, devised by Thomas Carlyle (1775-1881), does so. This construction solves the equation $x^{2}+b x+c=0$ for all real values of $b$ and $c$; in addition, it shows when solutions are nonreal.

- On graph paper, plot the points $A(0,1)$ and $B(-b, c)$.

- Bisect $\overline{A B}$ at $M$.

- Construct a circle with center $M$ and radius $\overline{A M}$.

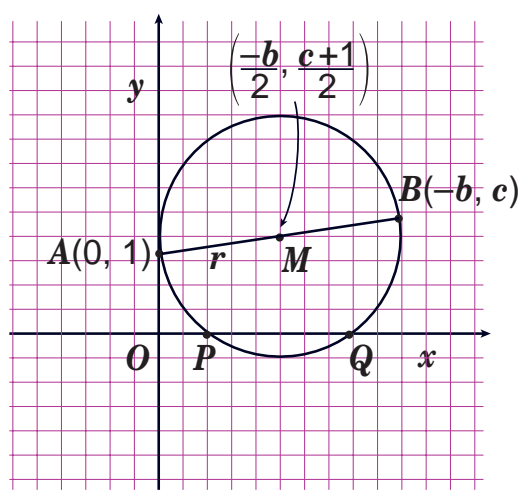

Fig. 11

Carlyle's method
- Label as $P$ and $Q$ the points where the circle intersects the $x$-axis.

\section{See figure 11.}

The directed lengths $O P$ and $O Q$ represent the solutions of the equation. The verification involves coordinate geometry that uses the facts that the circle has radius $A B / 2$; its center is the midpoint of $\overline{A B}$; and for any solution $x$ of the equation, $(x, 0)$ lies on the circle.

By choosing different values of $(-b, c)$, we can draw examples where the circle intersects the $x$-axis at two points, tangentially at one point, or not at all. These results will occur when the radius is, respectively, greater than, equal to, or less than the distance between the center of the circle and the $x$-axis, that is, $|c-1| / 2$.

The derivation of this condition, as well as its equivalence to the familiar test involving the sign of the discriminant $b^{2}-4 c$, may not be engaging to most second-year algebra students. However, students can experiment with different values of $b$ and $c$ and come to appreciate the relationship between the coefficients of a quadratic and the number of solutions. Since we began by dividing by $a$, the relationship really does involve all three coefficients.

This experiment can introduce a discussion of the discriminant, in which the precise nature of that relationship is developed.

\section{A justification of Carlyle's method}

If $r$ is the radius of the circle in figure 11, then $r=A B / 2$. Therefore,

$$
\begin{aligned}
r & =\frac{1}{2} \sqrt{(-b-0)^{2}+(c-1)^{2}} \\
& =\frac{\sqrt{b^{2}+(c-1)^{2}}}{2} .
\end{aligned}
$$

The center of the circle is the midpoint of $\overline{A B}$ :

$$
\left(\frac{-b}{2}, \frac{c+1}{2}\right) \text {. }
$$

If $(x, 0)$ is a point lying on the circle having this radius and center, then

$$
\begin{aligned}
\left(x-\frac{-b}{2}\right)^{2}+\left(0-\frac{c+1}{2}\right)^{2} & =r^{2}, \\
\left(x+\frac{b}{2}\right)^{2}+\left(\frac{c+1}{2}\right)^{2} & =\frac{b^{2}+(c-1)^{2}}{4}, \\
\left(x+\frac{b}{2}\right)^{2} & =\frac{b^{2}+(c-1)^{2}-(c+1)^{2}}{4}, \\
\left(x+\frac{b}{2}\right)^{2} & =\frac{b^{2}-4 c}{4}, \\
x^{2}+b x+\frac{b^{2}}{4} & =\frac{b^{2}}{4}-c, \\
x^{2}+b x+c & =0 .
\end{aligned}
$$

(Continued on page 319)
Students appreciate the relationship between the coefficients of a quadratic and the number of solutions 


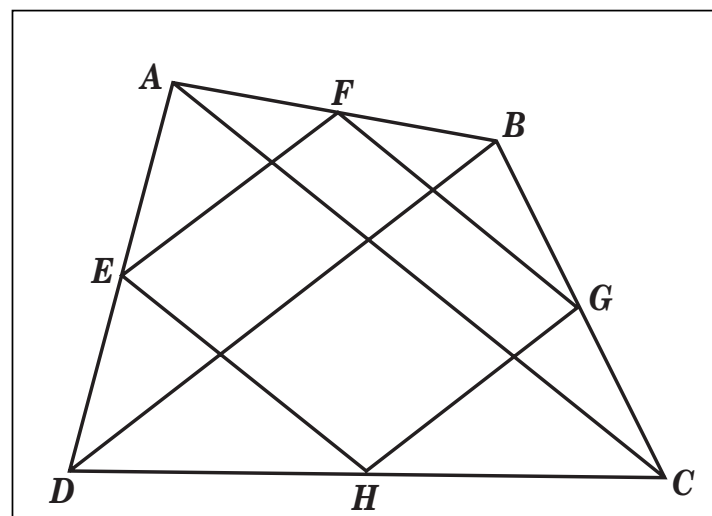

Fig. 4

Quadrilateral $A B C D$

meets the definition of a parallelogram (Varignon [1731] 1972, pp. 62-63).

When the Varignon parallelogram appears as a practice exercise in present-day geometry textbooks, students are sometimes asked to show that its opposite sides are congruent, each side having half the length of the diagonal to which it is parallel. However interesting these pairs of congruences may be, they are not essential for establishing that the figure is a parallelogram.

The parallelogram theorem gives evidence of the lucidity and craftsmanship of the gifted teacher for whom it is named.

A future article in the Mathematics Teacher will examine some implications of Varignon's theorem and suggest exercises for exploratory inquiry and deduction.

\section{BIBLIOGRAPHY}

Boyer, Carl B. A History of Mathematics. New York: John Wiley \& Sons, 1968.

Costabel, Pierre. "Varignon, Pierre." In Dictionary of Scientific Biography, vol. 13. New York: Charles Scribner's Sons, 1976.

Coxeter, Harold S. M., and Samuel L. Greitzer. Geometry Revisited. New York: Random House and L. W. Singer Co., 1967.

Guerlac, Henry. Newton on the Continent. Ithaca, N.Y. and London: Cornell University Press, 1981.

Rumyantsev, Valentin V. "Varignon Theorem." In Encyclopaedia of Mathematics, vol. 9. Dordrecht, Boston, London: Kluwer Academic Publishers, 1993.

Varignon, Pierre. Elemens de Mathematique de Monsieur Varignon. Paris: Pierre-Michel Brunet, Fils, 1731. New Canaan, Conn.: Readex Microprint Landmarks of Science Series, 1972.

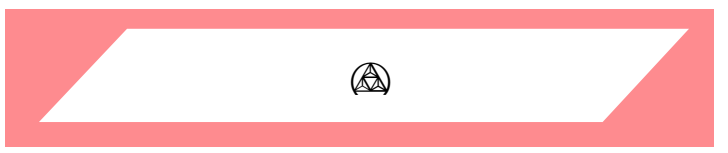

\section{Geometric Approaches to Quadratic Equations from Other Times and Places}

\author{
(Continued from page 313)
}

\section{BIBLIOGRAPHY}

Descartes, René. The Geometry of René Descartes. Translated by David Eugene Smith and Marcia L. Latham. New York: Dover Publications, 1954.

Eves, Howard. An Introduction to the History of Mathematics. Philadelphia: Saunders College Publishing, 1992.

Flax, Rosabel. "A Squeeze Play on Quadratic Equations." Mathematics Teacher 75 (February 1982): 132-34.

Heath, Thomas L. The Thirteen Books of Euclid's Elements. 2nd ed. New York: Dover Publications, 1956. Katz, Victor J. A History of Mathematics. Reading, Mass.: Addison Wesley Longman, 1998.

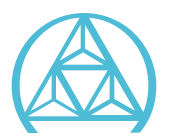

